\title{
Mechanical Analysis of Five Methods of Tibiotalar Arthrodesis Using Screw Fixation Technique: A Cadaveric Study
}

\author{
YeokPin Chua ${ }^{1}$, WengKong Low ${ }^{2}$, Rukmanikanthan Shanmugam ${ }^{3}$, Aik Saw ${ }^{4}$, Mark C Blackney ${ }^{5}$
}

\begin{abstract}
Background: Many different techniques for ankle arthrodesis have been described. Compression screws require the least dissection and can be done percutaneously, hence leading to faster healing. It was conventionally carried out by using half-threaded cancellous AO screws to achieve the compressive effect across the arthrodesis site. Even with the introduction of newer implants and screw systems, there is a lack of standardization in the surgical technique used in ankle arthrodesis surgery; and there is no recent study comparing the mechanical strength of these newer implants. Hence, we performed the study to determine the biomechanical properties of the five surgical techniques for ankle fusion as mentioned above.

Materials and methods: Fifteen fresh frozen cadaveric ankles were obtained and the specimens were then divided into five groups: three samples for each group; group I (two cross standard AO screw technique), group II (two cross headless compression screw technique), group III (two cross headless compression screws with anterior locking plate), group IV (three cross headless compression screws), and group V (IO Fix System). Arthrodesis fixation was then performed according to the groups divided. Each of the specimens was then tested following a standard loading protocol for its stiffness in plantar flexion, dorsiflexion, inversion, and eversion using Instron machine in NOCERAL PPUM. Results collected were analyzed using SPSS Ver 22.

Results: The results showed that group II has higher stiffness in all aspects when compared with group I. We also found that group III is the stiffest construct in the aspect of plantar flexion and dorsiflexion, and it is statistically significant when comparing plantar flexion stiffness with the group I $(p=0.042)$ and group II $(p=0.039)$. On the other hand, group IV is the stiffest construct inversion and eversion comparing to other groups. Group I is the weakest construct in all-loading aspects.

Conclusion: From the results of our study, we concluded that the newer generation of screws does have a biomechanical advantage over the conventional standard half-threaded AO screws. We recommend the use of headless compression screws in replacement of the standard AO half-threaded cancellous screws, and to use two cross screws with an anterior plate for open technique, or three screws fixation technique for arthroscopic ankle fusion.
\end{abstract}

Keywords: Ankle arthrodesis, Anterior plate, AO screw, Arthroscopic ankle fusion, Headless screw.

Journal of Foot and Ankle Surgery (Asia Pacific) (2021): 10.5005/jp-journals-10040-1179

\section{BACKGROUND}

Ankle arthritis is a common yet debilitating problem worldwide. Post-traumatic ankle arthritis is the leading cause of ankle arthritis. ${ }^{1}$ Other causes include ankle instability, inflammatory conditions, infection, recurrent hemarthrosis, neuropathic, and neoplastic conditions. ${ }^{1,2}$ Multiple operative choices of treatment are, but ankle arthrodesis remains the most widely accepted treatment for endstage ankle arthritis.,

Methods of ankle arthrodesis can be generally divided into an external fixation or internal fixation. Internal fixations are gaining more popularity in recent years, as studies have shown that they offer more stability at the talotibial arthrodesis site compared to external fixation, as reported by Mueckley et al. ${ }^{5}$

Compression screws require the least dissection and can be done percutaneously, hence leading to faster healing. This was conventionally carried out by using half-threaded cancellous $\mathrm{AO}$ screws to achieve the compressive effect across the arthrodesis site. However, headless compression screws have shown that they carry less complication rate, and higher compression effect compared with the conventional $\mathrm{AO}$ screws, ${ }^{6,7}$ and being low profile design where the entire screw head can be embedded into the cortex of the bone, reduce the complication of implant prominence. This unique
${ }^{1}$ Department of Orthopaedics (Foot and Ankle Orthopaedic Surgery), Institute of Medical Healthcare Development, Sunway University, Sunway Medical Centre, Selangor, Malaysia

${ }^{2-4}$ Department of Orthopaedic Surgery, National Orthopaedic Centre of Excellent for Research and Learning, University of Malaya, Kuala Lumpur, Malaysia

${ }^{5}$ Orthopaedic Department, The Park Clinic, St. Vincent's Private Hospital, East Melbourne, Melbourne, Australia

Corresponding Author: YeokPin Chua, Department of Orthopaedics (Foot and Ankle Orthopaedic Surgery), Institute of Medical Healthcare Development, Sunway University, Sunway Medical Centre, Selangor, Malaysia, Phone: +60193309245, e-mail: yeokpin1@yahoo.com

How to cite this article: Chua Y, Low W, Shanmugam R, et al. Mechanical Analysis of Five Methods of Tibiotalar Arthrodesis Using Screw Fixation Technique: A Cadaveric Study. J Foot Ankle Surg (Asia Pacific) 2021;8(4):201-205.

Source of support: Nil

Conflict of interest: None

property makes it ideal for fixation in subcutaneous areas, e.g., over the dorsum of the foot, and around the ankle region. Odutola et al. ${ }^{7}$ 
concluded that using a headless screw fixation for arthroscopic ankle arthrodesis prevents symptomatic metalwork prominence and the requirement for removal.

This study was thus conceptualized to determine if there is increased stiffness in constructs using the newer generation of screw system as compared to the conventional standard AO cancellous screws and to compare the stiffness between five different screw fixation constructs used in ankle fusion.

\section{Materials and Methods}

Fifteen human tibiotalar joints were harvested from 15 imported fresh frozen cadaveric lower limbs. Each harvested sample consisted of the talus and distal half of the tibia and all covering soft tissue was removed.

A planar cut of tibia plafond and talus dome was performed to create a flat surface for arthrodesis. To achieve uniform and comparable cut surfaces, the tibia plafond cut was done using Salto Total Ankle Replacement Mobile Version surgical instruments from Tornier Surgical Implants. The Salto Mobile Version cutting guide was attached to the tibia bone with pins. An alignment check was done to make sure that the guide places following the tibia bone's axis. Five millimeters of tibia plafond were resected using an oscillating saw through the cutting guide. This results in a cut surface that is orthogonal to the axis of the tibia. The foot is then placed at $90^{\circ}$ to the tibia, with manual compression of the talar dome onto the flattened cut surface of the tibia plafond. $3-5 \mathrm{~mm}$ of talar dome was resected using an oscillating saw, parallel to the cut surface of the tibia plafond. The cut surfaces were inspected to assure a flat and even cut surface. The specimens were then divided into five groups with three samples each for different ankle arthrodesis construct as shown in Figure 1.

Upon completion of screw placement across the tibiotalar arthrodesis site, the specimens were measured and cut at $6 \mathrm{~cm}$ proximal to the arthrodesis site. This was done to standardize the lever arm length during biomechanical testing later. Four short 1.4 $\mathrm{mm} \mathrm{K}$-wire were then driven into the talus bone (two on the talar head, and two at the body), to act as a scaffold to help anchor the small talus better into the resin.

A local engineering firm was engaged to aid in the design and fabrication of mounting jigs needed for this study. The jigs aimed to provide a stable and secure coupling of the specimens to the testing machine and allow conversion of the compression and extension loading of Instron Material Testing System (MTS) into plantar flexion, dorsiflexion, eversion, and inversion forces on the arthrodesis site.

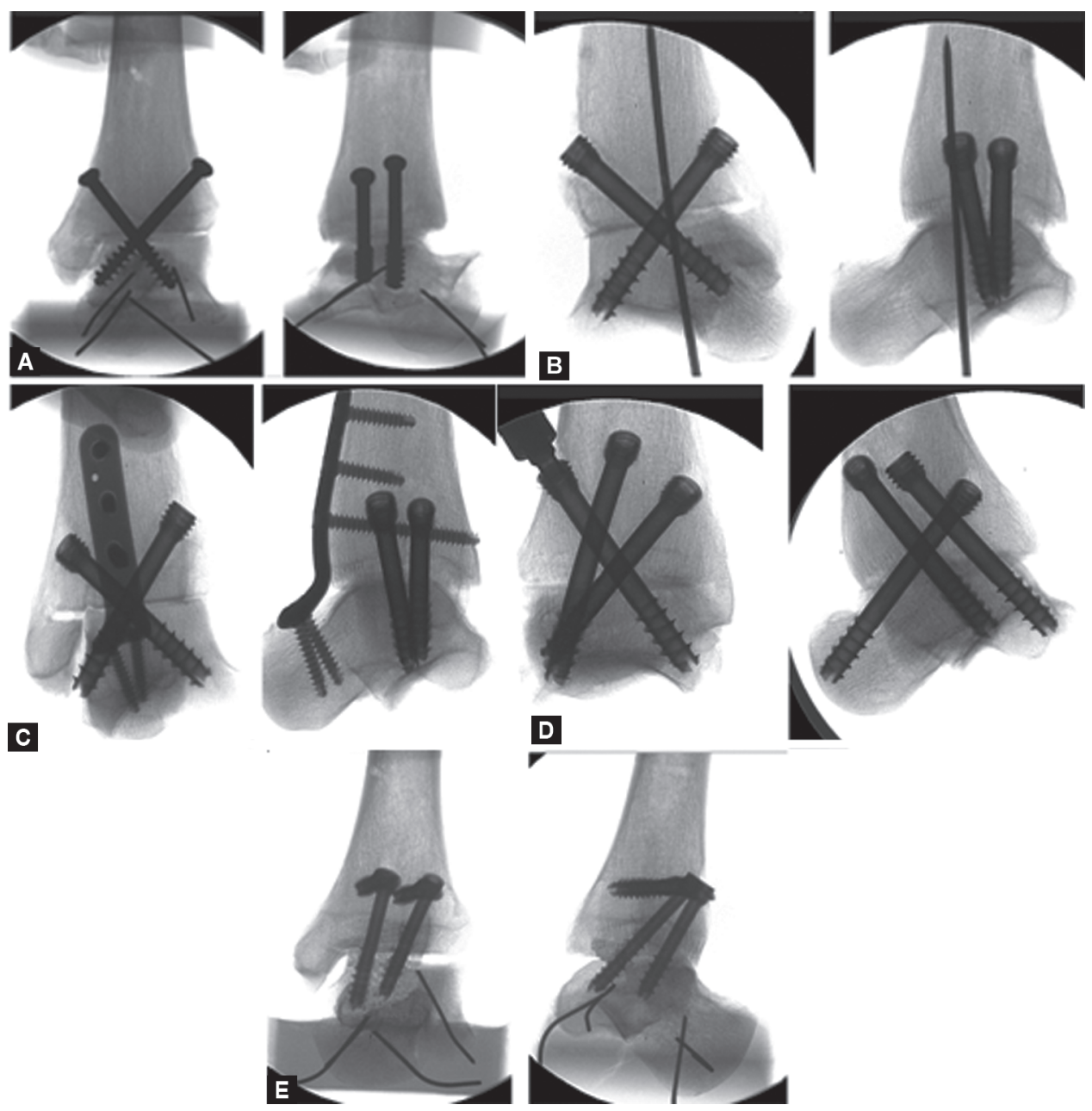

Figs $1 \mathrm{~A}$ to E: Example of radiograph on the bone sample after fixation: (A) Group I (two cross screws construct using AO $6.5 \mathrm{~mm}$ half-threaded cancellous screws); (B) Group II (two cross screws construct using Synthes $6.5 \mathrm{~mm}$ headless compression screws); (C) Group III (two cross screws construct using Synthes $6.5 \mathrm{~mm}$ headless compression screws with MetaFix Ankle Arthrodesis Plate by Merete); (D) Group IV (three screws construct using Synthes $6.5 \mathrm{~mm}$ headless compression screws using tripod fixation technique); (E) Group V (two Anterior IO Fix System Screws) 


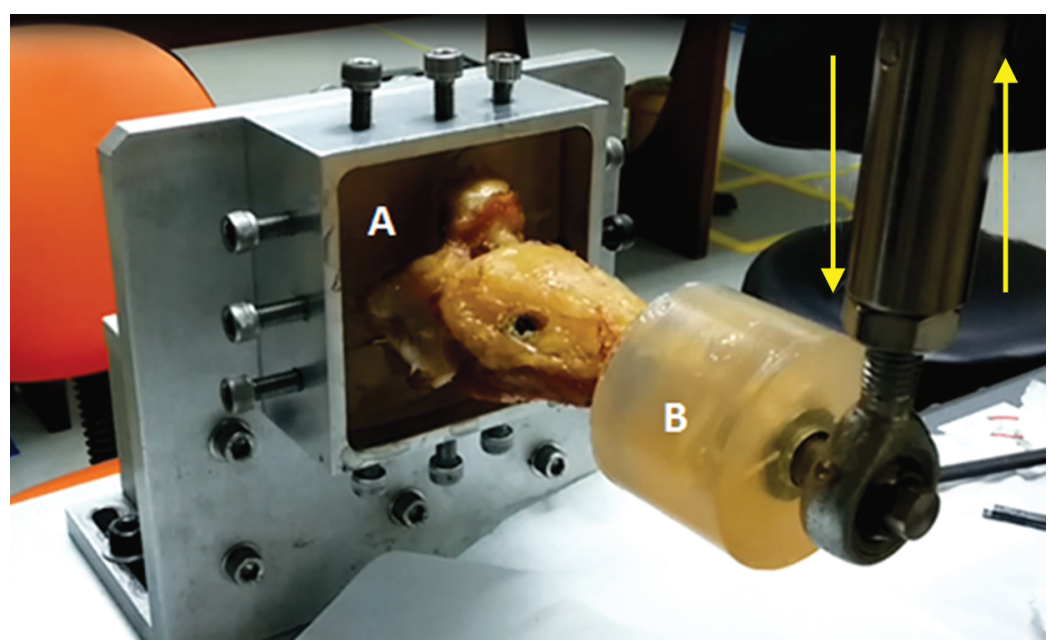

Figs $2 \mathrm{~A}$ and B: A specimen is mounted horizontally on the Instron machine. The talus end (A) is fixed, while the tibia end (B) is mobile

The mount has two components; the first component function to secure the proximal end of the distal tibia (Fig. 2B) to the lead cell of the Instron MTS. It has ball joint ring holder modularity which allows an arch movement of the specimen during the compression and extension loading, without causing a translational displacement of the specimen. The second component (Fig. 2A) functions to secure the talus end of the specimen to the base of the Instron machine and allow a motion-free coupling during the mechanical loading process.

Each of the specimens was then tested following a standard loading protocol for its stiffness in plantar flexion, dorsiflexion, inversion, and eversion using Instron machine in Biomechanical Testing Lab at the National Orthopaedic Centre for Research and Learning (NOCERAL), Department of Orthopaedic Surgery, Faculty of Medicine, University Malaya. Before the initiation of mechanical tests, the machine was calibrated by qualified engineers.

Results collected were analyzed using SPSS software (IBM SPSS Statistics Ver 22). The results were compared using the multifactorial ANOVA test, and correlations were checked between the stiffness and bone density, as well as between stiffness and contact surface area. $p$ value of $<0.05$ was considered significant.

\section{Results}

Each specimen was tested for its stiffness in plantar flexion, dorsiflexion, eversion, and inversion. The mean stiffness of each group was calculated and analyzed using the multifactorial ANOVA test. The summary of results is shown in Table 1.

\section{Plantar flexion}

Mechanical loading on the specimens to simulate plantar flexion was performed. The multifactorial ANOVA analysis of plantar flexion stiffness showed that there is a significant difference among the five groups. Further analysis using Tukey's post hoc test was done and it showed that group III was significantly stiffer than group I and group II with $p$ value 0.042 and 0.039 , respectively (Fig. 3).

There is no significant difference between group III and other groups.

\section{Dorsiflexion}

Similarly, for dorsiflexion testing, group III showed the highest stiffness followed by group IV and group III. Group I demonstrates the lowest stiffness among the five groups of the arthrodesis method (Fig. 4).
Table 1: Summary of mean stiffness of each group obtained from analysis by multifactorial ANOVA test for plantar flexion, dorsiflexion, eversion, and inversion

\begin{tabular}{|c|c|c|c|c|}
\hline & Group & Mean $(\mathrm{N} / \mathrm{m})$ & $\begin{array}{l}\text { Std deviation } \\
(\mathrm{N} / \mathrm{m})\end{array}$ & Sig. \\
\hline \multirow[t]{5}{*}{ Plantar flexion } & Group I & 7,192 & 4,222 & 0.03 \\
\hline & Group II & 9,299 & 4,904 & \\
\hline & Group III & 40,151 & 12,720 & \\
\hline & Group IV & 24,901 & 14,938 & \\
\hline & Group V & 18,668 & 20,559 & \\
\hline \multirow[t]{5}{*}{ Dorsiflexion } & Group I & 11,272 & 7,713 & 0.23 \\
\hline & Group II & 13,209 & 10,402 & \\
\hline & Group III & 35,494 & 20,977 & \\
\hline & Group IV & 20,812 & 10,185 & \\
\hline & Group V & 18,003 & 18,431 & \\
\hline \multirow[t]{5}{*}{ Eversion } & Group I & 8,624 & 5,098 & 0.39 \\
\hline & Group II & 12,298 & 6,664 & \\
\hline & Group III & 18,696 & 9,859 & \\
\hline & Group IV & 25,777 & 15,761 & \\
\hline & Group V & 16,401 & 15,926 & \\
\hline \multirow[t]{5}{*}{ Inversion } & Group I & 9,835 & 6,611 & 0.68 \\
\hline & Group II & 13,726 & 9,130 & \\
\hline & Group III & 19,176 & 9,087 & \\
\hline & Group IV & 21,209 & 9,160 & \\
\hline & Group V & 18,885 & 18,098 & \\
\hline
\end{tabular}

However, there is no statistically significant difference among the five groups when analyzed using the multifactorial ANOVA test ( $p$ value 0.23 ).

\section{Eversion}

Eversion and inversion loading showed slightly different stiffness patterns when compared with plantar flexion and dorsiflexion.

Group IV appeared to be the stiffest arthrodesis method in resisting eversion loading, followed by group III and group V. Group I has the lowest stiffness in resisting eversion loading (Fig. 5).

Nevertheless, there was no statistically significant difference between any of the five groups ( $p$ value 0.39). 


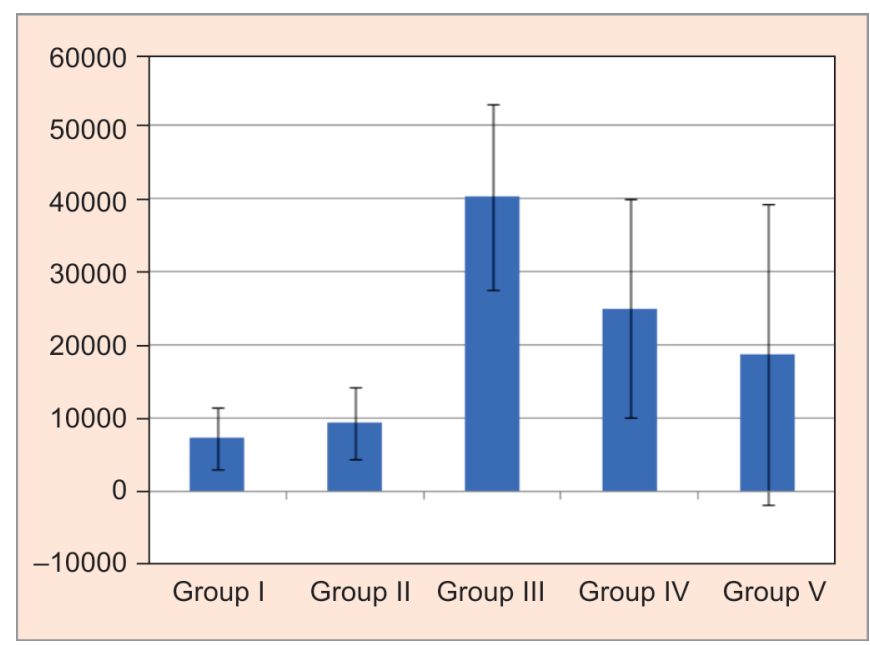

Fig. 3: Bar chart showing the plantar flexion stiffness of five groups of fixation

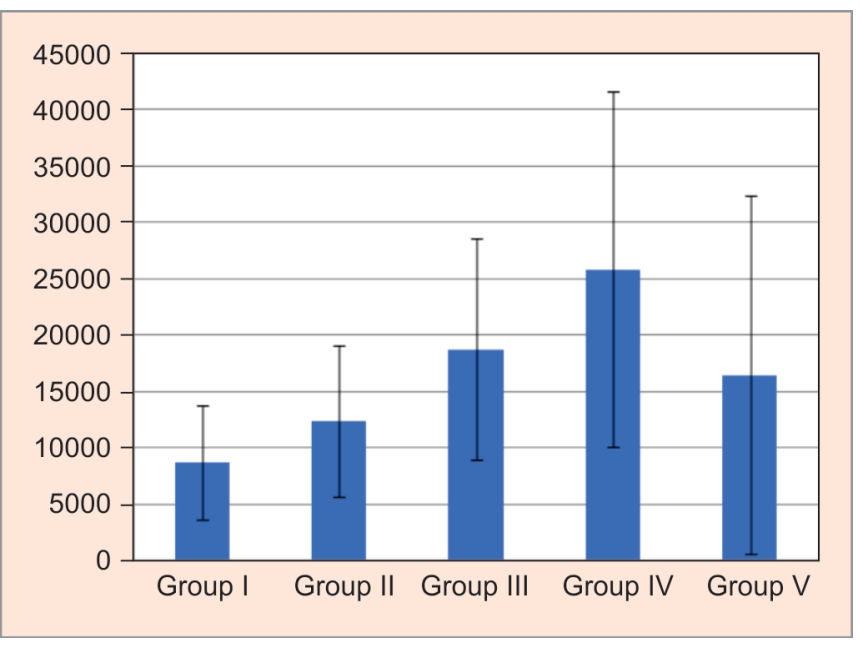

Fig. 5: Bar chart showing the eversion stiffness of five groups of fixation

\section{Inversion}

The stiffness pattern observed in inversion stiffness loading is similar to the eversion loading test, whereby group IV appeared to be the stiffest arthrodesis method, followed by group III and group V.Group I, again, has the lowest stiffness when compared with other groups (Fig. 6). Analysis using multifactorial ANOVA test showed no significant difference between the five groups ( $p$ value 0.68 ).

The results showed that group II has higher stiffness in all aspect when compared with group I. We also found that group III is the stiffest construct in the aspect of plantar flexion and dorsiflexion, and it is statistically significant while comparing plantar flexion stiffness with the group I ( $p=0.042)$ and group II $(p=0.039)$. On the other hand, group IV is the stiffest construct inversion and eversion comparing to other groups. Group I is the weakest construct in all-loading aspects.

\section{Discussion}

Delayed and failed fusion is a known complication following ankle arthrodesis. Reviews showed that the rate of successful fusion following ankle arthrodesis varied significantly. Some publications have even reported the rate of non-union to be as high as $35 \%{ }^{8}$

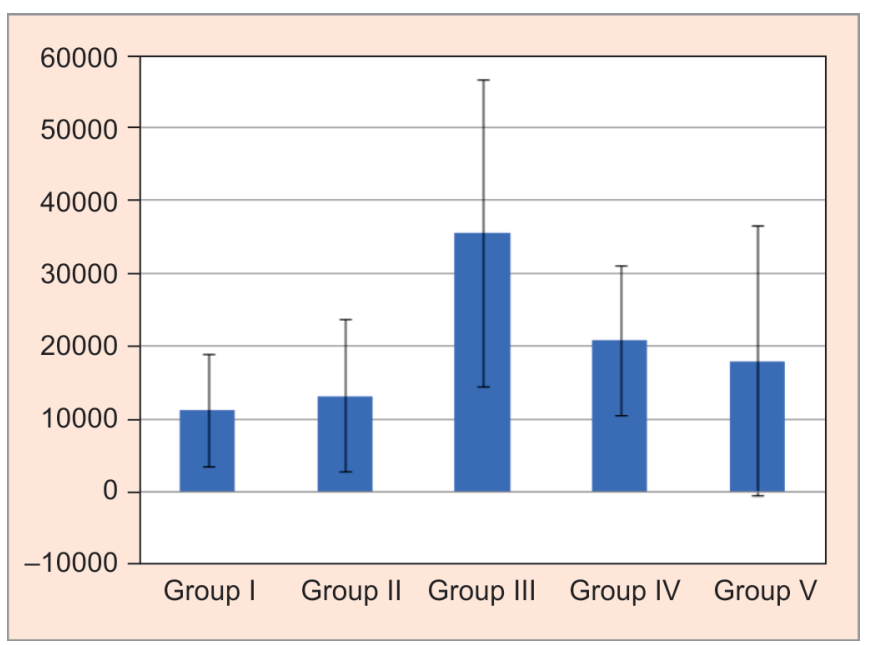

Fig. 4: Bar chart showing the dorsiflexion stiffness of five groups of fixation

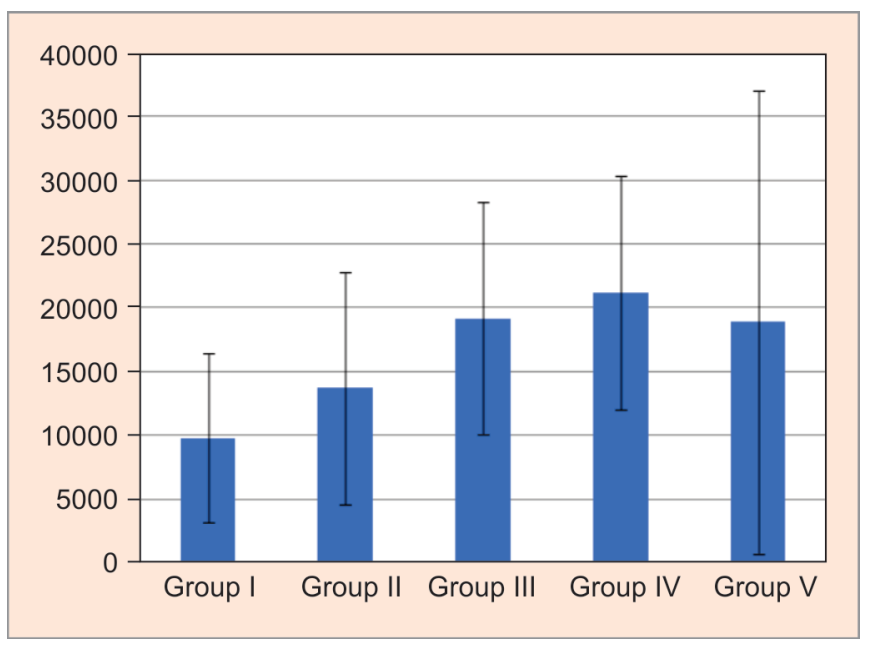

Fig. 6: Bar chart showing the inversion stiffness of five groups of fixation

In another small series by Dohm et al., ${ }^{9}$ he reported an even lower fusion rate of only $40 \%$. Factors affecting the union rate include surgical technique, as well as the type of patient.

There are $>30$ methods of fusion techniques reported to date ${ }^{8}$ and they can be divided into two large groups, namely, fusion by external fixation and fusion by internal fixation. Fusion using external fixation is limited mainly to cases with critical soft tissues condition and ongoing infection, ${ }^{10}$ where internally place implant imposes extra risks to the surgery. The techniques for internal fixation used include screws, angle blade plate, T plate, Wolf blade plate, intramedullary nail, and compression blade plate. ${ }^{10}$ Among all these, screw fixation has been found to achieve a better functional outcome and union rates, as was reported in various recent studies. ${ }^{11}$

Even using screw fixation techniques, there were many different constructs described, with the two cross screws technique being one of the most widely accepted screw fusion methods. Some authors use two parallel screws, three screws technique, two screws augment with anterior plate, and even four screws. To complicate the matter further, there are multiple new implants in the market used for ankle fusion, e.g., the headless compression screw to replace the standard AO screws, and IO fix IntraOsseous Fixation 
system (Extremity Medical) that advocates that their product can achieve better compression across the fusion site.

We found that there is a lack of standardization in the surgical technique used in ankle arthrodesis surgery. There is also no recent study in comparing the mechanical strength of these newer implants and assessing their superiority compared to the conventional AO screws that we have. Hence, we performed the study to determine the biomechanical properties of the five surgical techniques for ankle fusion as mentioned above. This is because various clinical and biomechanical trials showed that rigid internal fixation increases the rate of fusion of ankle arthrodesis. ${ }^{8}$

We found that two headless compression screws with anterior locking plate have the highest stiffness among all groups in dorsiflexion and plantar flexion loading, with the mean stiffness $40,151 \pm 12,720$ and $35,494 \pm 20,977 \mathrm{~N} / \mathrm{m}$, respectively. The plantar flexion stiffness is significantly stiffer than $2 \mathrm{AO}$ cross screws technique ( $p=0.41)$ and two headless compression cross screws technique $(p=0.39)$.

Three cross headless compression screws technique has the highest stiffness among all groups in eversion and inversion loading with the mean stiffness of 25,777 $\pm 15,761$ and 21,209 $\pm 9,160 \mathrm{~N} / \mathrm{m}$, respectively. However, this result was not statistically significant.

The conventional two AO cross screws construct has the lowest stiffness compared to other groups in all four aspects of load testing. This finding would justify the use of the newer generation of screws which are costlier.

In comparing two cross headless compression screws technique with and without anterior locking plate, we found that two cross HCS with an anterior locking plate is stiffer than only two cross HCS alone. This result outcome was expected as the construct is expected to be stiffer as we added fixation at a plane $90^{\circ}$ perpendicular to the cross screws. We found that two cross HCS with anterior plate increases the construct stiffness by a factor of 3.45 in sagittal modes, and 1.45 in coronal modes. These findings were consistent with results by Tarkin et al. ${ }^{12}$ reported an increase in sagittal mode stiffness by 3.5 times and coronal mode stiffness by 1.9 times while supplementing his three screws fusion construct with an anterior plate.

\section{Conclusion}

From the results of our study, we concluded that the newer generation of screws does have a biomechanical advantage over the conventional standard half-threaded AO screws. We recommend the use of headless compression screws in replacement of the standard AO half-threaded cancellous screws. To provide a more stable construct, two cross screws with an anterior plate technique is recommended for open method ankle arthrodesis and three screws technique is recommended for arthroscopic ankle fusion technique.

\section{References}

1. Saltzman CL, Salamon ML, Blanchard GM, et al. Epidemiology of ankle arthritis: report of a consecutive series of 639 patients from a tertiary orthopaedic center. lowa Orthop J 2005;25:44-46.

2. Di Stefano JG, Stephen Pinney M. Ankle arthritis: etiology and epidemiology. Seminars Arthrop 2010;21(4):218-222.

3. Bethany Gallagher M, Treatment for End-Stage Ankle Arthritis Re-evaluated.

4. DeHeer PA, Catoire SM, Taulman J, et al. Ankle arthrodesis: a literature review. Clin Podiatr Med Surg 2012;29(4):509-527. DOI: 10.1016/j. cpm.2012.07.001.

5. Mueckley TM, Eichorn S, von Oldenburg G, et al. Biomechanical evaluation of primary stiffness of tibiotalar arthrodesis with an intramedullary compression nail and four other fixation devices. Foot Ankle Int 2006;27(10):814-820. DOI: 10.1177/1071100706 02701011.

6. Danielle Chicano EA. Podiatry today:. A closer look at a versatile internal fixation device 2012;25(9):14-20.

7. Odutola AA, Sheridan BD, Kelly AJ. Headless compression screw fixation prevents symptomatic metalwork in arthroscopic ankle arthrodesis. Foot Ankle Surg 2012;18(2):111-113. DOI: 10.1016/j. fas.2011.03.013.

8. Rowan R, Davey KJ. Ankle arthrodesis using an anterior AO T plate. J Bone Joint Surg Br 1999;81(1):113-116. DOI: 10.1302/0301620x.81b1.8999.

9. Dohm M, Purdy BA, Benjamin J. Primary union of ankle arthrodesis: review of a single institution/multiple surgeon experience. Foot Ankle Int 1994;15(6):293-296. DOI: 10.1177/107110079401500602.

10. Nihal A, Gellman RE, Embil JM, et al. Ankle arthrodesis. Foot Ankle Surg 2008;14(1):1-10. DOI: 10.1016/j.fas.2007.08.004.

11. Zwipp H, Rammelt S, Endres T, et al. High union rates and function scores at midterm followup with ankle arthrodesis using a four screw technique. Clin Orthop Relat Res 2010;468(4):958-968. DOI: 10.1007/ s11999-009-1074-5.

12. Tarkin IS, Mormino MA, Clare MP, et al. Anterior plate supplementation increases ankle arthrodesis construct rigidity. Foot Ankle Int 2007;28(2):219-223. DOI: 10.3113/fai.2007. 0219. 